\title{
Symptom Prevalence and Burden in Cancer Patients with and without HIVIAIDS Reffered for Palliative Care
}

\author{
Ludoviko Zirimenya $^{1}$, Charles Musoke ${ }^{1}$ and Evelyn Hutt ${ }^{2}$ \\ 1. Hospice Africa Uganda, Hospice Africa Uganda, Makindye, Kampala, Uganda \\ 2. University of Colorado Health Science Center, Aurora, Colorado, USA
}

\begin{abstract}
The prevalence of cancer and that of HIV/AIDS is increasing in Uganda and throughout sub Saharan Africa. Unfortunately, little is known about the prevalence and burden of symptoms in patients with AIDS-Cancer and Cancer alone at first referral to a palliative care service. This study sets out to compare the prevalence and symptom burden between patients with AIDS-Cancer and those with Cancer only referred to a palliative care setting. Retrospective point prevalence survey of 150 randomly selected charts of patients referred to Hospice Africa Uganda (HAU) as per 2013. Of the 471 eligible patients' charts, 168 were randomly selected and only 150 were included in the study. A chart review instrument was used to extract information from the charts. Data were entered into Epidata version 3.1, cleaned and analysed using Epidata Analysis and Excel. Of the 150 patients' records: 78 (52\%) had Cancer only diagnosis and 72 (48\%) AIDS-Cancer diagnosis. Pain was most prevalent at $91.7 \%$ in the AIDS-Cancer group and $100 \%$ in the cancer only group. Three quarters reported pain as moderate to severe in both groups of patients. The five most prevalent symptoms in the AIDS-Cancer group were pain (91.7\%), social distresses (38.9\%), body swelling (27.8\%), Anorexia (22.2\%) and skin eruption (16.7\%) while in the Cancer alone group were pain (100\%), body swelling (25.6\%), Anorexia (23.1\%), social distresses (20.9\%) and fatigue (17.9\%). The average number of symptoms was 4.8 (2.3) in the Cancer group and 4.7 (2.3) in the AIDS-Cancer group Pain is highly prevalent in both Cancer only and AIDS-Cancer patients. The four most prevalent symptoms namely pain, social distresses, body swelling and anorexia are similar in both groups of patients. Social distresses occur highly in AIDS-Cancer patients.
\end{abstract}

\section{Acronyms}

$\begin{array}{ll}\text { AIDS } & \text { Acquired immunodeficiency syndrome } \\ \text { CVWs } & \text { Community Volunteer Workers } \\ \text { CMV } & \text { Cytomegalovirus } \\ \text { FRCP } & \text { Fellow in the Royal College of Physicians } \\ \text { GLOBOCAN } & \text { Global Cancer Network } \\ \text { HAART } & \text { Highly Active Anti-Retroviral Therapy } \\ \text { HAU } & \text { Hospice Africa Uganda } \\ \text { HAUREC } & \text { Hospice Africa Uganda Research and Ethics } \\ \text { HIV } & \text { Committee } \\ \text { MOH } & \text { Muman Immunodeficiency virus } \\ \text { NGO } & \text { Non-Governmental Organization } \\ \text { PC } & \text { Palliative Care } \\ \text { PLWHA } & \text { People Living With HIV/AIDS } \\ \text { PCP } & \text { Pneumocystis Carinii Pneumonia } \\ \text { SSA } & \text { Sub- Saharan Africa } \\ \text { UNAIDS } & \text { United Nations Programme on HIV/AIDS } \\ \text { WHO } & \text { World Health Organization } \\ \text { WPCA } & \text { Worldwide Palliative care Alliance }\end{array}$

Corresponding author: Ludoviko Zirimenya, research field: palliative medicine. E-mail: ludovikoz@yahoo.co.uk.

\section{Introduction}

\subsection{Need for Palliative Care in Africa}

According to WHO and Worldwide Palliative care Alliance (WPCA) publication, Global Atlas of Palliative Care at the End of Life, only 1 in 10 people who need palliative care - that is medical care offered to persons with life threatening illness to improve their quality of life by addressing their physical, psychological, social and spiritual distresses-are currently receiving it [1]. Palliative care according to WHO affirms life and regards dying as a normal process, neither hastens nor postpones death, provides relief from pain and other distressing symptoms, integrates the psychological and spiritual aspects of care, offers a support system to help patients live as actively as possible until death and offers a support system to help patients' families cope during the patient's illness and in their own bereavement [2]. 
Palliative care should not be used as an alternative but should be started alongside other interventions. The WHO recommends palliative care to start at the point of diagnosis of a potentially life limiting illness for the patient and his/her family throughout the disease trajectory, and after the death of the patient where after the family is bereaved. Early palliative care in cancer patients has been seen to greatly improve their quality of life, mood and it decreases the use of aggressive care at their end of life but still prolonging their survival [3].

In a recent review that assessed Africa's palliative care progress since 2005, Grant et al noted that a lot had been achieved in the past 10 years, including (1) integrating palliative care delivery into the national health systems of 28 out of 57 countries, (2) providing oral morphine, a cheap and strong analgesic in 48 countries, (3) an increase in post graduate and undergraduate palliative care courses in African Universities and (4) over 141 donors in Africa that are taking palliative care forward [4]. Despite these achievements, there is still a huge and unmet need for palliative care in low resource settings like Uganda. It is estimated that every year more than 20 million patients need palliative care at end of life of whom $80 \%$ are living in low and middle income countries [1].

\subsection{History of Palliative Care in Uganda}

The concept of Palliative care was introduced in Uganda in 1993 by Dr Anne Merriman FRCP through Hospice Africa Uganda with essential assistance and active collaboration of the Ministry of Health [5]. Uganda was the fourth country to commence palliative care in sub-Saharan Africa and first through HAU to commence it with a vision for the whole of Africa. The founding mission of HAU is to promote the relief of suffering in Africa through an affordable and cultural appropriate model. HAU incorporates as well 2 other sites namely Mobile Hospice Mbarara and Little Hospice Hoima. Together with clinical care service palliative care provision, HAU conducts education programs, both short and long courses, targeting health professionals in training and those in practice, equipping them with the skills and knowledge to practice palliative care.

Many palliative care developments have occurred in Uganda that include integrating palliative care into the Ministry of Health Five Year strategic plan as an essential clinical service, publication of policies and implementation guidelines to fast track access to PC and changing the legal statute to put in place palliative care specialists at the level of nurses and clinical officers to prescribe morphine [5].

\subsection{Burden of Cancer and HIV/AIDS in Africa}

In sub-Saharan African the most common life threatening illnesses reported by WHO are Cancer and HIV/AIDS. HIV/AIDs among heterosexuals was first described in Africa in 1982 [6]. To date $68 \%$ of all people living with HIV/AIDS reside in sub-Saharan Africa, although it has only $12 \%$ of the global population [7]. According to UNAIDS, despite recent reductions in the incidence of HIV/AIDS infections, its prevalence in settings like Uganda has continued to increase because people accessing antiretroviral therapy tend to live longer [7]. The current annual prevalence and incidence rates in Uganda stand at $6.4 \%$ and $0.95 \%$ respectively [8]. Despite antiretroviral therapy, the burden of symptoms in HIV/AIDS palliative care patients remains high in sub-Saharan Africa [9].

The occurrence of AIDS defining cancers and some non-AIDS defining cancer is increasing in Uganda [10]; non-AIDS defining cancers have been increasing in HIV/AIDS patients compared to the general population $[11,12]$. But little is known about the symptom burden of patients who have both HIV/AIDS and cancer. Most of these patients will ultimately need palliative care, which focuses on improving the quality of life of patients with life limiting illness [13].

The burden of cancer in sub-Saharan Africa continues to increase because of aging and adoption of cancer causing behaviors liking smoking and alcoholism [14]. In this region, HIV infection has also 
been linked to an increased risk of AIDS-defining cancers and a few non AIDS-defining cancers [10]. Infections in sub-Saharan African other than HIV/AIDS such as Hepatitis B have also been linked to cancers like Liver cancer in about $50 \%-100 \%$ of the cases [15].

According to the GLOBOCAN series report [16], an estimated 12.7 million new cancer cases and 7.6 million cancer deaths occurred in 2008 , with $56 \%$ of new cancers and $63 \%$ of the cancer deaths occurring in less developed regions in the world. Uganda has an annual cancer prevalence of $0.1 \%$, i.e. 22,000 new cases of cancer occur annually [5]. Cancer mortality rates are high, in part because of a poor health infrastructure and lack of basic access to oncological interventions like radiotherapy and chemotherapy.

In sub-Saharan Africa, cancer survival rates are the lowest globally. In Uganda for example, the 5 year survival does not exceed $13 \%$ for any cancer site except breast [17].

Cancer is a growing epidemic in Africa, although it is under recognized. It is estimated that 650,000 people of a population of 965 million are diagnosed annually with cancer [18]. Further-more there is an increased risk of dying from cancer in Africa when compared to developed countries. The most prevalent cancers in Africa are cervical cancer, breast cancer and Kaposis sarcoma [18], of which cervical cancer and Kaposis sarcoma's increased incidence has been linked to HIV/AIDS [19].

Each year about $0.5 \%$ of the total population in countries like Botswana, Ethiopia, Tanzania, Uganda and Zimbabwe die from HIV/AIDS or cancer [20], all these ultimately need palliative care contributing to the already high need of this service in sub-Saharan Africa [21].

\subsection{Symptoms in Cancer Patients}

Identifying symptoms helps in understanding the possible underlying cause and in formulating a management plan to use in the care of the terminally ill patients. Understanding of symptom prevalence and burden also helps in palliative care service provision and policy planning in terms of required resources like drugs, human resource and other logistics.

Globally, many factors have been shown to affect symptoms in cancer patients; age, gender and performance status [22]. Younger age, male gender and a poor performance status are associated with more symptoms in Cancer patients. Primary cancer site as well has been shown to affect symptoms. Head and neck, breast cancer and Cancer of Unknown Primary present with more symptoms compared to other Cancer sites [23]. In a Harding et al study done in South Africa and Uganda, being female and having a worse physical function were associated with a high symptom burden in palliative care HIV/AIDS patients in sub-Saharan Africa [24]. Symptoms in HIV/AIDS patients are due to the disease itself, opportunistic infections like tuberculosis, cryptococcal meningitis, PCP, CMV retinitis, and side effects of Highly Active Antiretroviral therapy.

Schuit et al. showed that symptoms are prevalent in cancer patients, with pain being reported by about $60 \%$ of the patients and $30 \%$ to $40 \%$ of all patients reporting constipation, nausea, loss of appetite, cough and dyspnea [25]. Pain as well is noted by the Everdingen et al study to be highly prevalent with a pooled prevalence of $>50 \%$ [26].

According to WHO, Cancer patients and their families have emotional, spiritual, social and economic consequences of cancer and its management. It recommends that it is the role of palliative care to address these needs in order to improve their quality of life and the ability to cope effectively.

Studies at HAU have looked at symptom burden and prevalence in HIV/AIDS patients [24] and in cancer patients [9], but not in those with both HIV/AIDS and cancer at point of referral to palliative care. These studies identified gender and functional status as contributing to the burden of symptoms in HIV/AIDS patients [24]; being female and having a worse 
physical function were associated with a higher symptom burden. Among the symptoms highlighted by Harding et al. to be most prevalent amongst cancer patients in sub-Saharan setting were pain (87.5\%), lack of energy (77.7\%), feeling sad (75.9\%), feeling drowsy (72.3\%) and worrying (69.9\%) [9]. In the Potter et al. study that looked at patients referred for palliative care, the five most prevalent symptoms were pain (64\%), anorexia (34\%), constipation (32\%), weakness (32\%) and dyspnea (31\%) [27].

According to Murray et al, Patients with cancer tend to experience similar trajectories in terms of their physical and social wellbeing during their illness: as their physical limitations increased, their social wellbeing declined [28]. This same study as well showed that as one nears end of life, the patient's main concerns are no longer physical but psychosocial in nature in terms of relations with other people. Though literature in our setting is scanty about social problems in cancer patients, other settings have highlighted social distresses in Cancer patients. In a study done by Wright et al, 6 categories of social problems were identified in Cancer patients: (1) managing in the home (2) health and welfare services (3) finances (4) employment (5) legal matters, (6) relationships. These social problems were as well related to high levels of problems psychologically [29].

Little is known about how symptoms in AIDS-Cancer patients compare with those of Cancer patients. For example, does HIV/AIDS in Cancer patients affect symptom prevalence and burden? Are there different symptoms affecting Cancer patients that are co-infected with AIDS?

This study sets out to understand how co-occurrence of HIV/AIDS and cancer affects symptom burden and prevalence in patients referred for palliative care services, in order to inform and improve palliative care practice.

\section{Research Design and Methods}

Research question addressed: How does the prevalence and burden of symptoms in HIV/AIDS-Cancer patients compare with those among Cancer patients referred for palliative care at HAU?

\subsection{Aims}

To determine how the prevalence of symptoms among HIV/AIDS-Cancer patients compares with that among Cancer only patients referred for palliative care at HAU.

To determine the average severity of reported pain among HIV/AIDS-Cancer patients and Cancer only patients referred for palliative care at HAU.

To determine how the symptom burden among HIV/AIDS-Cancer patients compares with that of Cancer only patients referred for palliative care at HAU.

\subsection{Methods}

\subsubsection{Study Design}

This was a retrospective, point prevalence medical records review study.

\subsubsection{Setting:}

The study was carried out at Hospice Africa Uganda (HAU) Kampala site, which was established in 1993 and offers total palliative care with an Outpatient clinic onsite, 3 outreach points in neighboring areas and home visit services to those that live within a $25 \mathrm{~km}$ radius of Kampala city center. This site attends to about 800 patients, both children and adults, in a year both from within and outside its catchment area like from other districts of Uganda and neighboring African countries.

\subsubsection{Target Population}

All palliative care patients with Cancer with and without HIV/AIDS in Uganda in 2013

\subsubsection{Accessible Population}

1. Patients' case notes with HIV/AIDS-Cancer on the HAU care programme in 2013

2. Patients' case notes with Cancer only on the HAU care programme in 2013

2.2.5 a Inclusion Criteria

Cancer only patients 1-95 years and 
Cancer-HIV/AIDS patients 1-95 years on HAU care programme in 2013.

\subsection{5 b Exclusion Criteria}

Charts that had more than $50 \%$ of data missing were excluded from the study.

\subsection{6 a Study Period}

The study charts considered where those of patients enrolled on Hospice Africa Uganda palliative care program between 1995 and 2013.

\subsection{6 b Sample Size}

The Kish and Leslie formula for calculating sample size in prevalence studies was used to estimate the required study sample size.

$$
\mathrm{N}=\mathrm{Z}^{\wedge} 2 \mathrm{pq} / \mathrm{e}^{\wedge} 2
$$

Where

$\mathrm{N}$ is the sample size

$\mathrm{Z}$ is the desired confidence interval

$P$ is the estimated proportion of an attribute

e is the desired level of precision

$\mathrm{q}$ is $1-\mathrm{p}$

Pain being the most prevalent symptom reported in available literature, was used as the attribute to calculate the required sample size. The Harding et al study done in our setting that looked at prevalence and burden of symptoms amongst cancer patients attending palliative care in two African countries; Pain was the most prevalent symptom at $87.5 \%$ hence the $P$ value of 0.875 .

$95 \%$ was the desired level of confidence used in the study.

$$
\begin{gathered}
\mathrm{N}=(1.96)^{\wedge} 2 * 0.875 * 0.125 /(0.05)^{\wedge} 2 \\
\mathrm{~N}=168
\end{gathered}
$$

Out of the 471 case charts, 168 were randomly selected using the stat trek software [30], but only 150 records were considered of which 78 had Cancer only diagnosis and 72 had HIV/AIDS-Cancer. 18 of the records were not considered as over $70 \%$ of the data in them were missing.

\subsubsection{Data Collection}

A potential list of symptoms was compiled onto a chart review instrument (appendix 1) basing on available research reports on symptom burden in similar settings $[9,24,31]$ and clinical experience. From available literature and clinical experience, an initial list of 54 symptoms inclusive of symptom groups like urinary symptoms that included dysuria, frequency and urgency and body swelling inclusive of facial, upper and lower limb swelling were used as in other studies of symptom prevalence [27]. Social distresses were all grouped as such on the chart review instrument and included financial constraints, lack of food, no family support, abandonment, denial of access to loved ones, lack of housing, lack of employment, conflicts with loved ones, failure to disclose to loved ones and other ill family members. Spiritual distresses were also all grouped as such on the chart review instrument and included fear of death, fear of the unknown, a sense of uncertainty, blame of self, feeling punished or judged by God, loss of sense of self or relationships or meaning. Sexuality distresses were as well grouped as such on the chart review instrument and included fears and concerns related to a changed body, new orifices or body parts, altered sensory patterns, altered libido and reduced sexual function. Pain score at initial presentation was as well noted on the list, this score was reported by a patient basing on a 5 point Numerical rating scale that has been validated as a pain measurement tool in a palliative care setting [32].

The numerical rating scales used for pain assessment by Hospice Africa Uganda are mainly two; namely the numerical hand rating scale and smiley faces as shown below:

Other demographic data that was included on the chart review instrument were age, sex, marital status, religion, occupation and referral source. The clinician's assessment of the patient's insight into illness was as well captured.

All data were collected retrospectively from the initial clinical assessment visit and documented onto a chart review instrument. This initial clinical assessment visit is normally conducted by a doctor, clinical officer 


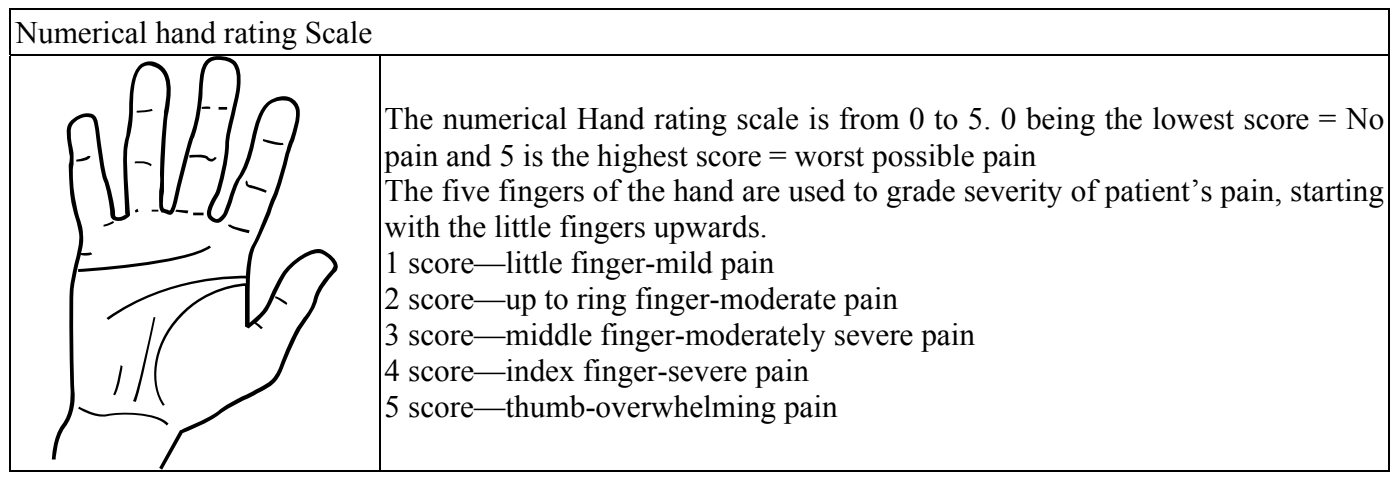

Scoring pain in children: this is only used when a child can recognize faces.

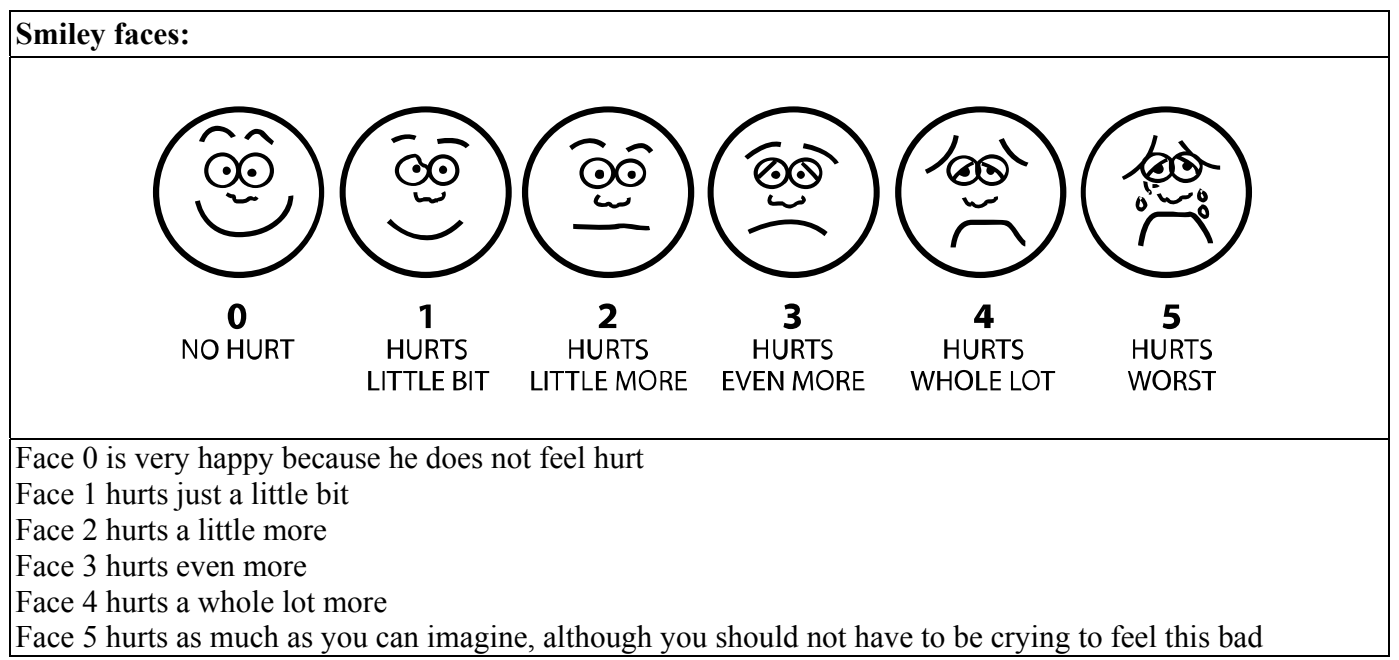

or a nurse using a structured patient's assessment form that is always completed on admission onto the Hospice Africa Uganda program. The initial visit is normally conducted on site in the clinic or in the patient's home or in the community outreaches depending on where the patient is seen. It is normally a consultation of approximately 2 hours where the clinician doing the assessment interviews the patient using the HAU intake form (appendix 3) as a guide to holistically address the issues and thereafter make a management care plan. A follow up schedule is always made within a week to check on how the patient and family are doing.

\subsubsection{Main Outcome Measures}

The main outcome of the study was the average number of symptoms in each group at the initial clinical assessment visit. The prevalence of symptoms among AIDS-Cancer patients and Cancer only patients was then computed. Pain severity score of patients with both HIV/AIDS and cancer compared to those with cancer only at the initial palliative care clinical assessment visit was the other outcome considered.

\subsubsection{Data Management and Analysis}

The data were entered into Epi Data version 3.1, backed and cleaned to prevent data loss. Statistical analysis was carried out using Epi Data analysis and Excel. Data analysis was done with the help of Mr. Handel Ian, the statistician provided by the university faculty with experience in medical research.

We first described the characteristics of the study population. Continuous variables were summarized in means (standard deviation) and median (inter quartile range). Categorical data was summarized using the frequency and the percentages and results are presented in tabulated form.

To address the first objective, the point prevalence of 
each symptom on the chart review instrument was calculated as: number of patients with symptom of interest divided by the total number of patients in that group of patients. This product was multiplied by $100 \%$ to get the symptom point prevalence. The unpaired (two-sample) t-test was used to compare point prevalence of each symptom between the two groups of patients.

To address the second objective, each patient's score was assigned under the respective category of pain severity that is; mild, moderate, severe and overwhelming. Their respective frequency and percentages per group was calculated and tabulated. The Mann-Whitney U test was used to check the significance of the difference in reported pain severity between the two groups

To address the third objective, the total number symptoms as per initial clinical assessment visit were totaled for each patient, and then the mean and standard deviation for each group of patients was calculated. The unpaired (two sample) $t$ test was used to compare the symptom burden between the two groups of patients.

The acceptable level of significance used throughout the analysis was $P<0.05$ with a $95 \%$ confidence interval.

Interpretation of the results and the discussion of the study objectives were done by the principal investigator with the help of the supervisors.

\subsection{Quality Control}

To ensure internal validity of the study, the following measures were undertaken:

The chart review instrument was pretested, standardized and piloted before the study commenced. Initially 15 charts were piloted, and the chart review instrument had to be modified to capture other symptoms that were not initially included. Some variables like age, sex and subject number were lacking on the form but where later incorporated into it.

The chart review instrument was checked and edited for completeness at the end of each data extraction from the file.

An independent reviewer randomly sampled at least 5 charts and re-extracted the data to verify their quality.

\subsection{Ethical Considerations}

Approval was obtained from the faculty of the University of Edinburgh and Hospice Africa Uganda Institutional Review Committee (HAU-IRC). As this was a retrospective review and patients' identifiers were left out, exemption from informed consent was also obtained from the HAU research and ethics committees.

\section{Results (Presentation of Findings)}

\subsection{Sample Characteristics}

Out of the 417 eligible charts, a total of 150 patients' records were studied (Table 1): 78 (52\%) had Cancer only and 72 (48\%) had both AIDS and Cancer. 18 out of the 168 targeted records were excluded from the study as $70 \%$ of the data of interest were missing in them.

The patients' age in the Cancer only group ranged from 2 years to 95 years with a mean age of 50.3 years $(\mathrm{SD}=4.04)$. The patients' age in the AIDS-Cancer group ranged from 1 year to 61years with a mean age of 38.2 years $(\mathrm{SD}=0.707)$. Majority of patients in both groups were above 18 years; 73 of the $78(93.6 \%)$ in the Cancer only group and 67 of the $72(93.1 \%)$ in the AIDS-Cancer group. The majority as well in both groups were female at $63 \%$ in the Cancer only group and $65 \%$ in the AIDS-Cancer group.

Among the total 150 referrals used in this study, $49 \%$ were Health workers' referral, $34 \%$ were self-referrals, and $15 \%$ where Community Volunteer workers' referrals. The majority of referrals to palliative care came from Health workers at $50 \%$ in the cancer only group and $48.6 \%$ in the AIDS-Cancer group. This is followed by self-referrals at $38.5 \%$ in the Cancer only group and $29.2 \%$ in the AIDS-Cancer group with fewest referrals in both groups coming from 
Table 1 Patients' characteristics.

\begin{tabular}{llll}
\hline Variable & Cancer group $(\mathbf{n}=\mathbf{7 8})$ & $\begin{array}{l}\text { HIV/AIDS and Cancer group } \\
(\mathbf{n}=\mathbf{7 2})\end{array}$ & $\boldsymbol{P}$ value \\
\hline Mean age (SD) & $53.3(19.5)$ & $38.2(12.6)$ & $<0.00001$ \\
\hline Gender & & $32(35 \%)$ & $\mathbf{0 . 3 6 5}$ \\
\hline Male & $29(37 \%)$ & $40(65 \%)$ & \\
Female & $49(63 \%)$ & & $\mathbf{0 . 2 6 5}$ \\
\hline Oncology intervention & & $42(58.3 \%)$ & \\
YES & $53(67.9 \%)$ & $29(40.3 \%)$ & $\mathbf{0 . 0 7 3 4}$ \\
NO & $25(32.1 \%)$ & & \\
\hline Type of referral & & $35(48.6 \%)$ & \\
Health worker & $39(50 \%)$ & $16(22.2 \%)$ & $21(29.2 \%)$ \\
CVW & $7(9 \%)$ & & \\
Self & $30(38.5 \%)$ & &
\end{tabular}

the community volunteer workers (CVWs). The CVWs are community people recommended by community elders and trained by HAU to identify people that are in need of palliative care services in their communities and then link them to HAU for care. They also help HAU follow up patients already enrolled on its program in their respective communities. In both groups of patients, CVW referrals were more in the AIDS-Cancer group at $22.2 \%$ compared to $9 \%$ in the Cancer only group.

$67.9 \%$ of cancer only patients and $58.3 \%$ of AIDS-Cancer patients had an oncology intervention. These interventions included chemotherapy, radiotherapy, surgery and hormonal therapy for hormonal responsive cancers like breast cancer and prostate cancer.

All persons in our study that had HIV/AIDS were enrolled in ART centres that provided comprehensive HIV/AIDS care as per the World Health Organization guidelines. Our data shows that $79.2 \%$ had already started HAART at the time of their palliative care enrollment, though we did not look at CD4 counts to see if those not on HAART where eligible for it.

The 3 commonest cancers in the Cancer group were breast $(23.1 \%)$, gastrointestinal malignancies (oesophagus, stomach, liver, gastro intestinal Stroma, colorectal, anal) at $21.8 \%$ and prostate $(7.7 \%)$ while in the AIDS-Cancer group the most common cancers were Kaposis Sarcoma (45.8\%), cervix (27.8\%) and gastrointestinal malignancies (11.1\%). A further breakdown of the different cancer types among the patients enrolled on the study is in appendix 2 .

\subsection{Prevalence of Symptoms among HIV/AIDS-Cancer Patients and Cancer only Patients}

The five most prevalent symptoms in the Cancer only group of patients were pain at $100 \%$, body swelling at $25.6 \%$, anorexia at $23.1 \%$, social distress at $20.5 \%$ and fatigue at $17.9 \%$, whereas in the HIV/AIDS-Cancer patients they were pain at $91.7 \%$, social distress at $38.9 \%$, body swelling at $27.8 \%$, anorexia at $22.2 \%$ and skin eruption at $16.7 \%$. Among these five most prevalent symptoms, only four that is-pain, body swelling, social distress and anorexia-were common to both groups of patients; whereas skin eruption was more in AIDS/Cancer group and fatigue was more in the cancer only group.

Though fatigue was not among the five most prevalent in the AIDS-Cancer patients, it stood at a prevalence of $13.9 \%$. As well skin eruption was not among the most prevalent in the Cancer patients and stood at a prevalence of $3.8 \%$. Of all the symptoms, prevalence difference was only statistically significant in 4 symptoms namely; social distresses, skin eruption and skin itching in the AIDs-Cancer group and pain in the Cancer only group of patients.

The least prevalent physical symptoms in the AIDS-Cancer group were tremors $(1.4 \%)$, anger 
(1.4\%), bleeding (1.4\%), disorientation (1.4\%), yellow eyes $(1.4 \%)$, per rectal discharge $(1.4 \%)$ and per rectal bleeding (1.4\%). In the Cancer group, the least prevalent physical symptoms were dry mouth $(1.3 \%)$, yellow eyes $(1.3 \%)$, haematuria $(1.3 \%)$, flatulence (1.3\%), hiccups $(1.3 \%)$, per vaginal bleeding $(1.3 \%)$, prolapsed colostomy $(1.3 \%)$, memory loss $(1.3 \%)$, confusion (1.3\%), palpitations (1.3\%) and disfigurement $(1.3 \%)$. These symptoms were totally different in the two groups.

The physical symptoms that were completely absent in the AIDS-Cancer group were disfigurement, prolapsed colostomy, palpitation, confusion, alopecia, halitosis, hiccups, flatulence, haematuria and dry mouth. In the Cancer group, the physical symptoms that were not reported by the patients were vaginal sores, disorientation, tremors, and incontinence.

The psychological distresses like anger and low mood though present were not prevalent and stood at $1.4 \%$ and $5.6 \%$ in the HIV/AIDS-Cancer group and $0 \%$ and $2.6 \%$ in the Cancer only group respectively. In the AIDS-Cancer group, no patient reported worry and while in the Cancer only group, no patient reported anger.

The sexuality issues stood at $2.8 \%$ in the
HIV/AIDS-Cancer group and at $1.3 \%$ in the Cancer group.

The social distresses which included financial constraints, lack of food, no family support, abandonment, denied access to loved ones, lack of housing, lack of employment, conflicts with loved ones, failure to disclose to loved ones and the burden of other ill family members were more prevalent in the AIDS-Cancer patients at $38.9 \%$ compared to $20.5 \%$ in the Cancer only group of patients. This difference in prevalence was statistically significant at a $p$ value of 0.0151 .

Detail regarding the social distresses in each group of patients is summarized in the table 3 below.

As noted in the table above, the most reported social distress in both groups of patients was financial constraints at $53.6 \%$ in the HIV/AIDS-Cancer patients and $50 \%$ in the Cancer only patients. The trends of social distresses across both groups of patients were similar as shown by the prevalence of the most commonly occurring 3 social distresses of financial constraints, abandonment and limited family support. Issues related to disclosure of illness to loved ones occurred only in the AIDS-Cancer group of patients and were at a prevalence of $10.7 \%$. Though some

Table 2 Patients' cancer diagnosis.

\begin{tabular}{lll}
\hline Tumor type & Cancer only group $\mathbf{N}=\mathbf{7 8}$ & AIDS-Cancer group $\mathbf{N}=\mathbf{7 2}$ \\
\hline Head and neck & $6(7.7 \%)$ & $1(1.4 \%)$ \\
Breast & $18(23.1 \%)$ & $1(1.4 \%)$ \\
Liver & $4(5.1 \%)$ & $1(1.4 \%)$ \\
Cervix & $7(9 \%)$ & $20(27.8 \%)$ \\
Prostate & $6(7.7 \%)$ & 0 \\
Kaposis & $2(2.6 \%)$ & $33(45.8 \%)$ \\
Skin & $3(3.8 \%)$ & $1(1.4 \%)$ \\
Multiple myeloma & $3(3.8 \%)$ & 0 \\
Lymphoma & $2(2.6 \%)$ & $3(4.2 \%)$ \\
Vulva & 0 & $2(2.8 \%)$ \\
Eye & $2(2.6 \%)$ & $1(1.4 \%)$ \\
Penis & $2(2.6 \%)$ & 0 \\
Ovary & $1(1.3 \%)$ & 0 \\
Sarcoma & $5(6.4 \%)$ & 0 \\
Gastrointestinal Tumors & $17(21.8 \%)$ & $8(11.1 \%)$ \\
Unspecified primary & 0 & $1(1.4 \%)$ \\
\hline A & & 0
\end{tabular}

A breakdown of the different types of malignancy among the patients enrolled on the study. 
Table 3 The prevalence of different symptoms in AIDS-Cancer and Cancer only patients.

\begin{tabular}{|c|c|c|c|c|}
\hline \multirow{2}{*}{ Symptom } & \multicolumn{2}{|c|}{ Prevalence of symptom } & \multirow{2}{*}{-P value } & \multirow{2}{*}{ OR(Confidence interval) } \\
\hline & AIDS and Cancer $n=72$ & Cancer only $\mathrm{N}=78$ & & \\
\hline Pain & $66(91.7 \%)$ & $78(100 \%)$ & 0.0093 & $0.06(0-1.18)$ \\
\hline Social distress & $28(38.9 \%)$ & $16(20.5 \%)$ & 0.0151 & $2.68(1.19-6.03)$ \\
\hline Body Swelling & $20(27.8 \%)$ & $20(25.6 \%)$ & 0.77 & $1.12(0.54-2.3)$ \\
\hline Anorexia & $16(22.2 \%)$ & $18(23.1 \%)$ & 0.9006 & $0.95(0.44-2.05)$ \\
\hline Skin eruption & $12(16.7 \%)$ & $3(3.8 \%)$ & 0.0089 & $5(1.35-18.53)$ \\
\hline Constipation & $11(15.3 \%)$ & $13(16.7 \%)$ & 0.816 & $0.9(0.38-2.16)$ \\
\hline Cough & $10(13.9 \%)$ & $11(14.1 \%)$ & 0.9699 & $0.98(0.39-2.47)$ \\
\hline Skin itching & $10(13.9 \%)$ & $3(3.8 \%)$ & 0.029 & $4.03(1.06-15.3)$ \\
\hline Skin ulcer & $11(15.3 \%)$ & $10(12.8 \%)$ & 0.6648 & $1.23(0.49-3.09)$ \\
\hline Fatigue & $10(13.9 \%)$ & $14(17.9 \%)$ & 0.0928 & $0.15(0.01-2.93)$ \\
\hline Abdominal distension & $8(11.1 \%)$ & $11(14.1 \%)$ & 0.5821 & $0.76(0.29-2.02)$ \\
\hline Vomiting & $9(12.5 \%)$ & $7(9 \%)$ & 0.4846 & $1.45(0.51-4.12)$ \\
\hline Per vaginal discharge & $8(11.1 \%)$ & $5(6.4 \%)$ & 0.3066 & $1.82(0.57-5.86)$ \\
\hline Immobility & $8(11.1 \%)$ & $5(6.4 \%)$ & 0.3066 & $1.82(0.57-5.86)$ \\
\hline Body discharge & $7(9.7 \%)$ & $4(5.1 \%)$ & 0.2809 & $1.99(0.56-7.12)$ \\
\hline Drowsiness & $7(9.7 \%)$ & $4(5.1 \%)$ & 0.2809 & $1.99(0.56-7.12)$ \\
\hline Diarrhea & $5(6.9 \%)$ & $4(5.1 \%)$ & 0.6398 & $1.38(0.36-5.36)$ \\
\hline Decreased activities of living & $5(6.9 \%)$ & $5(6.4 \%)$ & 0.8957 & $1.09(0.3-3.93)$ \\
\hline PV Bleeding & $5(6.9 \%)$ & $1(1.3 \%)$ & 0.077 & $5.75(0.66-50.42)$ \\
\hline Dysphagia & $4(5.6 \%)$ & $8(10.3 \%)$ & 0.289 & $0.52(0.15-1.79)$ \\
\hline Urinary symptoms & $4(5.6 \%)$ & $9(11.5 \%)$ & 0.1932 & $0.45(0.13-1.53)$ \\
\hline Low mood & $4(5.6 \%)$ & $2(2.6 \%)$ & 0.3503 & $2.23(0.4-12.59)$ \\
\hline Weight loss & $3(4.2 \%)$ & $6(7.7 \%)$ & 0.3687 & $0.52(0.13-2.17)$ \\
\hline Anxiety & $3(4.2 \%)$ & $1(1.3 \%)$ & 0.2733 & $3.35(0.34-32.94)$ \\
\hline Insomnia & $2(2.8 \%)$ & $4(5.1 \%)$ & 0.463 & $0.53(0.09-2.98)$ \\
\hline Sexuality issues & $2(2.8 \%)$ & $1(1.3 \%)$ & 0.5133 & $2.2(0.2-24.8)$ \\
\hline Fever & $3(4.2 \%)$ & $5(6.4 \%)$ & 0.5412 & $0.64(0.15-2.76)$ \\
\hline Nausea & $3(4.2 \%)$ & $3(3.8 \%)$ & 0.9203 & $1.09(0.21-5.57)$ \\
\hline Sore mouth & $3(4.2 \%)$ & $4(5.1 \%)$ & 0.7803 & $0.8(0.17-3.72)$ \\
\hline Increased salivation & $2(2.8 \%)$ & $3(3.8 \%)$ & 0.7157 & $0.71(0.12-4.4)$ \\
\hline Breathlessness & $2(2.8 \%)$ & $8(10.3 \%)$ & 0.0666 & $0.25(0.05-1.22)$ \\
\hline PR Discharge & $1(1.4 \%)$ & $2(2.6 \%)$ & 0.6075 & $0.54(0.05-6.03)$ \\
\hline PR Bleeding & $1(1.4 \%)$ & $2(2.6 \%)$ & 0.607 & $0.54(0.05-6.03)$ \\
\hline Bleeding & $1(1.4 \%)$ & $2(2.6 \%)$ & 0.6075 & $0.54(0.05-6.03)$ \\
\hline Memory loss & $1(1.4 \%)$ & $1(1.3 \%)$ & 0.9545 & $1.08(0.07-17.67)$ \\
\hline Anger & $1(1.4 \%)$ & 0 & 0.2963 & $3.29(0.13-82.16)$ \\
\hline Tremors & $1(1.4 \%)$ & 0 & 0.2963 & $3.29(0.13-82.16)$ \\
\hline Memory loss & $1(1.4 \%)$ & $1(1.3 \%)$ & 0.9545 & $1.08(0.07-17.67)$ \\
\hline Yellow eyes & $1(1.4 \%)$ & $1(1.3 \%)$ & 0.9545 & $1.08(0.07-17.67)$ \\
\hline Vaginal sores & $1(1.4 \%)$ & 0 & 0.2963 & $3.29(0.13-82.16)$ \\
\hline Disorientation & $1(1.4 \%)$ & 0 & 0.2963 & $3.29(0.13-82.16)$ \\
\hline Incontinence & $1(1.4 \%)$ & 0 & 0.2963 & $3.29(0.13-82.16)$ \\
\hline Halitosis & 0 & $2(2.6 \%)$ & 0.1713 & $0.21(0.01-4.47)$ \\
\hline Haematuria & 0 & $1(1.3 \%)$ & 0.3351 & $0.36(0.01-8.89)$ \\
\hline Flatulence & 0 & $1(1.3 \%)$ & 0.3351 & $0.36(0.01-8.89)$ \\
\hline Hiccups & 0 & $1(1.3 \%)$ & 0.3351 & $0.36(0.01-8.89)$ \\
\hline Alopecia & 0 & $3(3.8 \%)$ & 0.0928 & $0.15(0.01-2.93)$ \\
\hline Prolapsed colostomy & 0 & $1(1.3 \%)$ & 0.3351 & $0.36(0.01-8.89)$ \\
\hline Palpitation & 0 & $1(1.3 \%)$ & 0.3351 & $0.36(0.01-8.89)$ \\
\hline Worry & 0 & $1(1.3 \%)$ & 0.3351 & $0.36(0.01-8.89)$ \\
\hline Disfigurement & 0 & $1(1.3 \%)$ & 0.3351 & $0.36(0.01-8.89)$ \\
\hline Confusion & 0 & $1(1.3 \%)$ & 0.3351 & $0.36(0.01-8.89)$ \\
\hline Dry mouth & 0 & $1(1.3 \%)$ & 0.3351 & $0.36(0.01-8.89)$ \\
\hline Spiritual distress & 0 & 0 & & \\
\hline
\end{tabular}


patients reported social issues, they declined to disclose them on the initial assessment and requested the clinician to give them time to think about it. Patients that made such request were about $3.6 \%$ in the AIDS-Cancer patients and $6.3 \%$ in the Cancer only group of patients.

Spiritual distresses were not identified in either group of patients.

\subsection{Severity of Reported Pain among}

HIV/AIDS-Cancer and Cancer only Patient

Pain is highly prevalent in both groups of patients as shown in Table 2, all (100\%) cancer only patients presented with pain, while $91.7 \%$ of the AIDS-Cancer patients had pain. In the HIV/AIDS-Cancer group 11\% presented with no pain, $9.7 \%$ with mild pain, $27.8 \%$ with moderate pain and $45.8 \%$ with severe pain while in the Cancer only group 3.8\% presented with no pain, $9 \%$ with mild pain, $21.8 \%$ with moderate pain and $55.1 \%$ with severe pain. The $\mathrm{p}$ value was not statistically significant at 1 .

\subsection{Symptom Burden among HIV/AIDS-Cancer Patients and Cancer only Patients}

The mean number of symptoms per patient across both groups was $4.8(\mathrm{SD}=2.3)$. In the AIDS-Cancer group it was $4.7(\mathrm{SD}=2.3)$ and in the Cancer only group $4.8(\mathrm{SD}=2.3)$, which is not significantly different $(P=0.5)$ (Table 5)

\subsection{Comparison of Sub Groups}

In general the HIV/AIDS-Cancer group was much younger than the Cancer only group. The most common cancer is affected by the presence of HIV/AIDS: in the

Table 4 Social distresses among AIDS-Cancer and Cancer only patients.

\begin{tabular}{llll}
\hline & $\begin{array}{l}\text { HIV/AIDS and Cancer } \\
\text { patients }(\mathbf{N}=\mathbf{2 8})\end{array}$ & $\begin{array}{l}\text { Cancer only patients } \\
(\mathbf{N = 1 6 )}\end{array}$ & $\boldsymbol{P}$ value \\
\hline Financial constraints & $15(53.6 \%)$ & $8(50 \%)$ & 0.072 \\
Lack of employment & $1(3.6 \%)$ & 0 & 0.296 \\
Abandonment & $3(10.7 \%)$ & $3(18.8 \%)$ & 0.92 \\
Conflicts with loved ones & $1(3.6 \%)$ & 0 & 0.296 \\
Not disclosing to loved ones about the illness & $3(10.7 \%)$ & 0 & 0.0686 \\
Limited family support & $3(10.7 \%)$ & $1(6.3 \%)$ & 0.273 \\
Other ill family members & $1(3.6 \%)$ & 0 & 0.296 \\
Not going to school & 0 & $1(6.3 \%)$ & 0.335 \\
Not able to have children & 0 & $1(6.3 \%)$ & 0.335 \\
Undisclosed social issues & $1(3.6 \%)$ & $2(6.3 \%)$ & 0.608 \\
TOTAL & $28(100 \%)$ & $16(100 \%)$ & \\
\hline
\end{tabular}

Table 5 The burden of pain severity among AIDS-Cancer and Cancer patients.

\begin{tabular}{lll}
\hline Pain severity & HIV/AIDS and Cancer & Cancer \\
\hline No pain & $8(11 \%)$ & $3(3.8 \%)$ \\
Mild pain (1-2) & $7(9.7 \%)$ & $7(9 \%)$ \\
Moderate pain (3) & $20(27.8 \%)$ & $17(21.8 \%)$ \\
Severe pain (4-5) & $33(45.8 \%)$ & $43(55.1 \%)$ \\
Data missing & $4(5.7 \%)$ & $8(10.3 \%)$ \\
\hline
\end{tabular}

Table 6 Symptom burden per patient group.

\begin{tabular}{ll}
\hline & Mean number of symptoms per patient(SD) \\
\hline All & $4.8(2.3)$ \\
HIV/AIDS and cancer & $4.7(2.3)$ \\
Cancer & $4.8(2.3)$ \\
\hline
\end{tabular}


AIDS-Cancer group it was Kaposis Sarcoma, an AIDS defining Cancer, at $45.8 \%$, while in the Cancer only group it was breast cancer at $23.1 \%$.

Comparison of symptom burden and prevalence by diagnosis showed that pain is the most prevalent symptom in both groups. Of the five most prevalent symptoms in both groups of patients, 4 are similar and included pain, body swelling, social distress and anorexia.

Breathlessness, urinary symptoms and dysphagia were more prevalent in the Cancer only group of patients compared to the AIDS-Cancer group of patients at $10.3 \%$ vs. $2.8 \%, 11.6 \%$ vs. $5.6 \%$ and $5.6 \%$ vs. $10.3 \%$ respectively. Other symptoms were equally prevalent in both AIDS-Cancer patients and Cancer only patients; these were cough at $13.9 \%$ vs. $14.1 \%$, skin ulcer at $15.3 \%$ vs. $12.8 \%$, abdominal distension at $11.1 \%$ vs. $14.1 \%$, and vomiting at $12.5 \%$ vs. $9 \%$ respectively. Vaginal discharge, skin itching and skin eruption were more prevalent in the AIDS-Cancer patients compared to the Cancer only patients at $11.1 \%$ vs. $6.4 \%, 13.9 \%$ vs. $3.8 \%$ and $16.7 \%$ vs. $3.8 \%$ respectively.

\section{Discussion of Findings}

Some of demographics of Cancer persons accessing palliative care was similar between those with AIDS-Cancer and those with Cancer only, except that AIDS-Cancer patients were significantly younger, (mean age of 38.2 years vs. 53.3 years in those with cancer only). In terms of sex, the majority of patients in both groups were female at $63 \%$ in the Cancer only group and $65 \%$ in the AIDS-Cancer group of patients. Our study thus confirms earlier work of Harding et al which showed a mean age of 36.5 years and $73.2 \%$ of 224 patients were female in those with HIV/AIDS [24] and a mean age of 56.6 years and $64.3 \%$ of 112 patients were female in Cancer patients attending palliative care services [9].

There was a significant difference in the age and type of malignancy between the two groups of patients with the Cancer only group having much higher mean age of 53.3 years compared to 38.2 years in the AIDS-Cancer group. This age difference correlates well with the nature of cancers per group and their associated risk factors. The most prevalent Cancers in the Cancer only group were Breast at $23.1 \%$ and prostate at $7.7 \%$, which occur as one gets older and this is clearly manifested in the different mean age which was at 53.3 years in the Cancer only group and 38.2 years in the AIDS-Cancer group. The most prevalent cancers in the HIV/AIDS-Cancer group were cervical cancer at $27.8 \%$ and Kaposis sarcoma at $45.8 \%$, which are classified by WHO as AIDS defining cancers. There was no significant difference in sex between the two groups of patients.

Among the total 150 referrals used in this study, $49 \%$ were health workers' referral, $34 \%$ were self-referrals, and $15 \%$ where community volunteer workers' referrals. There were more CVW referrals for HIV/AIDS-Cancer patients at $22.2 \%$ compared to $9 \%$ in the Cancer only group. This we think is contributed by an increase in the community based strategies led by the Ministry of Health in combating HIV/AIDS, which has increased access to palliative care in the community. Existence of late referrals in some settings to palliative care is documented [33], there are several contributing barriers to access and referral to palliative care services inclusive but not limited to lack of knowledge and education among the health care and social care professionals and a lack of standardized referral criteria [34]. The CVW model as shown in our study contributes to increased referrals as these Community Volunteer workers are trained in identifying patients in need of palliative care and hence link them up to the services, creating a standardized referral criteria.

\subsection{Prevalence of Symptoms among HIV/AIDS-Cancer and Cancer Only Patient}

In our study, the five most prevalent symptoms in the AIDS-Cancer group were pain at $91.7 \%$, social 
distress at $30.6 \%$, body swelling at $27.8 \%$, anorexia at $22.2 \%$ and skin eruption at $16.7 \%$ while in the Cancer only group of patients they were pain at $100 \%$, body swelling at $25.6 \%$, anorexia at $23.1 \%$, social distress at $20.5 \%$ and fatigue at $17.9 \%$. These are different from those reported in the Harding et al studies done in the same setting [9, 24], where the five most prevalent symptoms were pain (82.6\%), feeling sad (75.4\%), feeling drowsy (74.1\%), worrying (73.2\%) and lack of energy (71.9\%) in 224 HIV/AIDS patients and pain (87.5\%), lack of energy (77.7\%), feeling $\operatorname{sad}(75.9 \%)$, feeling drowsy (72.3\%) and worrying (69.6\%) in 112 cancer patients. we believe this difference is due to the different study methodology employed in the Harding et al studies and our study. The Harding et al studies used seven days occurrence of symptoms, while ours used one day symptom occurrence to gauge their prevalence.

Among the top 5 most prevalent symptoms, 4 were similar in both groups; pain, body swelling, anorexia and social distress. This affirms an earlier conclusion by Harding et al [24] and Solano et al [35] that the prevalence of the most common symptoms in palliative care is the same across Cancer and HIV/AIDS groups of patients.

Apart from pain, the other symptoms do not tally with those in the Harding et al study. This may be attributed to the retrospective nature of our study that can lead to patients' records lacking some information and its accuracy wanting, or is it may be a new finding that will need to be ascertained further by future prospective research. Further it is not possible to ascertain whether all symptoms presented by the patients were recorded in their charts.

Though psychological problems are present in our sample at approximately $1 \%$, they are not among the top five most prevalent problems as reported in the Harding et al studies that reported them to be among the top 5 most prevalent symptoms at about $70 \%$. Our findings are similar to Potter et al study findings [27] that as well did not note psychological problems among the 5 most prevalent symptoms. Two possible explanations for this discrepancy are that our study looked at the point of referral to a palliative care setting while the Harding et al study focused on patients already in care. It is also possible that the different methodology they used of directly interviewing the patients, or that the clinical assessment chart used at HAU does not adequately assess psychological stress could explain some of the difference in findings. Further research could examine the symptom profiles of palliative care patients from the point of enrollment into care to the point of death.

Social distresses were more prevalent among AIDS-Cancer patients than cancer only patients (38.9\% vs. $20.5 \%$ ). This is comparable to the Wright et al study [29] that reported a prevalence of social problems in cancer patients attending palliative care at $30 \%$. The nature of social distresses among AIDS-Cancer and Cancer only patients, were similar to the 6 categories of social problems that were found by Wright et al to occur in Cancer patients. These categories were (1) managing in the home (2) health and welfare services (3) finances (4) employment (5) legal matters, (6) relationships [29]. Of these 6 categories, our study highlighted problems mainly in 2 categories, relationships and finances. The level of development in our setting being low predisposes the patients to high levels of poverty which explains why the financial problems ranked high in our study.

The difference in our study between the two groups in terms of AIDS-Cancer patients failing to disclose to their families about their illness could be explained by the stigma that is associated with AIDS compared to Cancer only diagnosis. AIDS related stigma is a well known fact in areas of both high and low prevalence rates $[36,37]$. This ranges from stigmatizing attitudes like blaming PLWHA for becoming infected with HIV, 'othering' or social distancing from the infected, disgust towards the symptoms caused by the disease, which all lead to negative stigmatizing behaviors like social isolation, gossiping and public shaming of 
PLWHA [37]. The long duration as well that the patients stay with the illness either Cancer only or AIDS-Cancer predisposes them to social distresses as carers are overwhelmed with amount of time required to give the patient. As well we speculate that as HIV/AIDS-Cancer patients are much younger than cancer only patients, their social situations like raising children, achieving their life goals is not yet achieved so hence the more social distress in them. Our study is likely among the few that has documented prevalence of social distresses in our setting. The Harding et al studies focused mostly on physical and psychological problems.

In our study, no spiritual distresses were reported in both groups of patients, in contrast to Moadel et al. study that showed presence of spiritual needs between $25 \%$ and $51 \%$ of 248 patients [38]. The presence of spiritual distresses in palliative care is also reported in the Selman et al study that was done in South Africa and Uganda among 285 patients that showed presence of these distresses in $21.4-57.9 \%$ of palliative care patients [39]. It is likely that our clinicians are more skilled at noting physical and social than spiritual distresses. It is also possible that referral to hospice ameliorates spiritual distress, such that patients do not report it as often, or that spiritual distress is shared by the patient only later in the course of their relationship with hospice clinicians. Further research would help clarify the cause of these discrepancies.

Sexuality distresses were reported in our study at $2.8 \%$ in the HIV/AIDS-Cancer group and $1.3 \%$ in the Cancer only group, which are much lower than that reported in the Harding et al study [9] that showed their presence in $21.4 \%$ of 112 patients. This markedly reduced level could be explained by clinicians' lack of skill in eliciting sexuality issues, as shown by the Lemieux et al study, which showed that only one participant out of seven had ever had a discussion or been asked by a health worker about sexuality issues [40]. There is as well a lot of misunderstanding by the health workers about their patients' sexuality which makes them tend to limit their inquiries about it to fertility, contraception, menopause, erectile status or capacity for intercourse [41-43].

Of all the symptoms reported by patients in the study, difference in prevalence was only statistically significant in four symptoms namely social distresses, skin eruption and skin itching in the AIDS-Cancer group and pain in the Cancer only group. This fact can be explained by the clinical nature of HIV/AIDS as it tends to predispose patients to opportunistic infections like tuberculosis, pneumonias, fungal infections and skin manifestations as shown in the WHO clinical staging of HIV/AIDS.

\subsection{Severity of Reported Pain among} HIV/AIDS-Cancer Patients and Cancer Only Patients

Our data reveal a higher prevalence of pain in both groups of patients when compared with earlier researches done in AIDS and cancer groups. Overall across the two groups, $96 \%$ of all the patients both AIDS-Cancer and Cancer only reported pain at the point of initial assessment. The prevalence of pain in our study was over $90 \%$ in both groups of patients. This is higher than that reported in the Schuit et al. study that found pain in $68 \%$ of 228 patients [25], the Potter et al. study that found it in $64 \%$ of 400 patients referred for palliative care [27], and the Van den Beuken-van Everdingen et al systematic review of 52 articles that showed a prevalence rate of $64 \%$ in cancer patients with advanced disease [26]. The variability in the prevalence of pain reported in our study we think is due to different study designs employed in all the studies. For example our study was retrospective in nature whereas the potter et al and the Schuit et al studies were prospective which could have eliminated some of the measurement bias that our study had due to the way the different clinicians documented information in the patients' files. As well the Van den Beuken-van Everdingen et al study was a systematic review with very little chances of bias and confounding affecting its results. But despite this, our study confirms findings of 
these studies that pain is highly prevalent in cancer patients.

Severity of pain varied little between the two groups, in the AIDS-Cancer group, $73.6 \%$ reported moderate to severe pain while in the Cancer only group $76.9 \%$ reported moderate to severe pain. Overall, $75 \%$ of all patients reported moderate to severe pain, which tallies with the review done by Van den Beuken-van Everdingen et al. that showed that more than $75 \%$ of cancer patients that reported pain graded it as moderate or severe [26]. In all studies reviewed, pain is reported as severe at $23.2 \%$ in the Harding et al studies, this is comparable to findings in our study that show $45.8 \%$ in the HIV/AIDS-Cancer group and $55.1 \% \%$ in the cancer group reported pain as severe. This goes to affirm that pain is prevalent and does occur commonly in patients referred for palliative care. It is likely that most patients referred for palliative care come especially because of uncontrollable pain. If one assumes that cancer patients with other, non-pain, uncontrolled physical, psycho-social and spiritual distresses are not being referred for palliative care, are they dying with these distresses or are their needs being met at their primary centres of care?

\subsection{Symptom Burden among HIV/AIDS-Cancer Patients and Cancer only Patients}

The symptom burden per patient was lower in our study at $4.7(\mathrm{SD}=2.3)$ in the HIV/AIDS-Cancer group and $4.8(\mathrm{SD}=2.3)$ in the Cancer only group in contrast to the Harding's studies that reported a higher mean number of symptoms over a seven day period of 18.1 $(\mathrm{SD}=6.9)$ in those with HIV/AIDS and $18(\mathrm{SD}=6.6)$ in those with cancer. This can be explained by difference in study designs. For example, our study was a one day symptom point-prevalence study where as the Harding studies were a seven day period prevalence studies. Harding's studies and ours reported little difference in symptom burden between HIV/AIDS and Cancer patients.

The symptom burden among the HIV/AIDS-Cancer patients and the Cancer only patients was not affected overall by the presence of HIV/AIDS as shown in Table 3, the mean number of symptoms overall was the same at $4.8(\mathrm{SD}=2.3)$. This is difficult to explain but we think that as $79.2 \%$ of HIV/AIDS patients were on HAART, this could have controlled AIDS related distresses that would have increased the symptom burden. This assumption is supported by the Brechtl et al study that showed that HAART in advanced AIDS patients significantly improves some clinical markers like weight, CD4 cell count and viral load so hence decreases on the symptom burden [44].

The clinical importance of our findings is that pain is highly prevalent in Cancer patients with or without HIV/AIDS and a majority of these patients report it as moderate to severe. The spectrum of symptom burden of Cancer patients is not affected by AIDS. Social distresses, skin eruption and skin itching are more prevalent in AIDS-Cancer patients and pain in the Cancer only patients, so palliative care practitioners should be on the lookout for them. As well symptom occurrence in terms of prevalence and burden is high and similar in both groups of patients and these symptoms are not only physical but also psychosocial in nature.

\section{Conclusions}

We have been able to estimate and compare the prevalence and burdens of symptoms in AIDS-Cancer patients and cancer only patients referred for palliative care to HAU. Pain is the most prevalent symptom in both AIDS-Cancer and Cancer only patients and the majority of patients in both groups reported their pain as Moderate to severe. Four of the 5 most prevalent symptoms in AIDS-Cancer and Cancer patients were similar and included pain, body swelling, social distress and anorexia. Among the AIDS-Cancer group, social distresses like abandonment, lack of money, lack of food, denied access to loved ones, lack of housing, lack of employment, conflicts with loved ones, failure to disclose to loved ones and the burden of other ill 
family members occurred highly and were the second and fourth most prevalent symptom in Cancer only patients. Psychological and sexuality distresses though present in both groups of patients are not reported to be highly prevalent. Though we were not able to identify any spiritual distresses in either groups of patients, another study is under way that will be able to identify, quantify and manage spiritual distresses using an approved well-validated tool called Spirit 8 [39]. Of all the symptoms, AIDS-Cancer patients were more statistically prone to presenting with social distresses, skin itching and skin eruption while Cancer only patients were more likely to present with pain. The symptom burden in both groups of patients at point of referral was similar and was not affected by the presence of AIDS. Though HIV infections in some populations has been shown to affect quality of life of patients [45], our study has shown that it does not affect the symptom prevalence and burden of patients at point of referral to a palliative care setting. This we think is due to the increasing access to comprehensive HIV/AIDS care through the available health structure under the Ugandan Ministry of Health.

Though by our study method we could not establish a causal relationship between the HIV/AIDS-Cancer on symptom prevalence and burden, we have been able to show that these patients present with a high burden of symptoms that are not just physical but also include psychosocial in nature.

A number of limitations impacted our study. First the study's retrospective and cross sectional nature made it impossible to draw causal relationships to symptom burden. Second about 3\% of the data of interest was missing from the patients' records. Third reliance on patients' records data instead of collecting new primary data may have introduced inaccuracies and biases such as lack of ability of the patient to report or convey distress over spiritual or sexual matters and lack of the clinicians' ability to fully elicit all the presenting problems of the patient or poorly documenting them in his/her chart.
In a palliative care setting, in addition to impeccable assessment of all cancer patients, cancer patients with HIV/AIDS should be keenly assessed for skin eruption, skin itching and any social problems, and where possible social work services should receive a higher priority, so that those in need may access them. We add our voice to earlier recommendations that clinicians should regularly assess their patients holistically and not focus only on physical symptoms, as shown that psycho social issues are also present in Cancer patients with or without HIV/AIDS.

\section{Acknowledgments}

I would like to acknowledge the support from The University of Edinburgh faculty who supported and mentored me through my studies that resulted in the successful completion of this Dissertation project. My research supervisors (the co-authors), staff of Hospice Africa Uganda and the patients whose data was used in this research, last but not least to my family and God. Thank you all very much.

\section{References}

[1] Connor, S., and Sepulveda, C. 2014. Global atlas of palliative care at the end of life. World Health Organization, Worldwide Palliative Care Alliance.

[2] O'Neill, B., and Fallon, M. 1997. "ABC of Palliative Care. Principles of Palliative Care and Pain Control." BMJ: British Medical Journal 315 (7111): 801.

[3] Temel, J. S., et al. 2010. "Early Palliative Care for Patients with Metastatic Non-small-cell Llung Cancer." New England Journal of Medicine 363 (8): 733-42.

[4] Grant, L., et al. 2011. "Palliative Care in Africa since 2005: Good Progress, but much Further to Go." BMJ Supportive \& Palliative Care 1 (2): 118-22.

[5] Jagwe, J. G., and Barnard, D. 2002. "The Introduction of Palliative Care in Uganda." Journal of palliative medicine 5 (1): 159-63.

[6] Serwadda, D., et al. 1985. "Slim Disease: a New Disease in Uganda and its Association with HTLV-III Infection." The Lancet 326 (8460): 849-52.

[7] Who, U. and Unicef, Global HIV/AIDS response: epidemic update and health sector progress towards universal access. Progress report. November, 2011.

[8] Hladik, W., et al. 2008. "The Estimated Burden of HIV/AIDS in Uganda, 2005-2010.” AIDS 22 (4): 503-10. 
[9] Harding, R., et al. 2011. "The Prevalence and Burden of Symptoms amongst Cancer Patients attending Palliative Care in two African Countries." European Journal of Cancer 47 (1): 51-6.

[10] Mbulaiteye, S.M., et al. 2006. "Spectrum of Cancers among HIV-infected Persons in Africa: The Uganda AIDS-Cancer Registry Match Study.” International journal of cancer 118 (4): 985-90.

[11] Shiels, M.S., et al. 2009. "A Meta-analysis of the Incidence of non-AIDS Cancers in HIV-infected Individuals." Journal of acquired immune deficiency syndromes 52 (5): 611.

[12] Shiels, M.S., et al. 2011. "Cancer Burden in the HIV-infected Population in the United States." Journal of the National Cancer Institute 103 (9): 753-62.

[13] Kaasa, S., and Loge, J. H. 2003. "Quality of Life in Palliative Care: Principles and Practice." Palliative Medicine 17 (1): 11-20.

[14] Jemal, A., et al. 2011. "Global Cancer Statistics." CA: a cancer journal for clinicians 61 (2): 69-90.

[15] Henry, S. H., Bosch, F. X., and Bowers, J. 2002. Aflatoxin, hepatitis and worldwide liver cancer risks, in Mycotoxins and Food Safety, Springer. p. 229-233.

[16] Ferlay, J., et al. 2010. "Estimates of Worldwide Burden of Cancer in 2008: GLOBOCAN 2008." International journal of cancer 127 (12): 2893-917.

[17] Sankaranarayanan, R., et al. 2010. "Cancer Survival in Africa, Asia, and Central America: a Population-based Study." The lancet oncology 11 (2): 165-73.

[18] Parkin, D. M., et al. 2008. "Part I: Cancer in Indigenous Africans - Burden, Distribution, and Trends." The lancet oncology 9 (7): 683-92.

[19] Parkin, D. M., et al. 1999. "AIDS-related Cancers in Africa: Maturation of the Epidemic in Uganda." AIDS 13 (18): 2563-70.

[20] Sepulveda, C., et al. 2003. "Quality Care at the end of Life in Africa." BMJ: British Medical Journal 327 (7408): 209.

[21] Harding, R., and Higginson, I. J. 2005. "Palliative Care in Sub-Saharan Africa.” The Lancet 36(9475): 1971-7.

[22] Walsh, D., Donnelly, S., and Rybicki, L. 2000. "The Symptoms of Advanced Cancer: Relationship to Age, Gender, and Performance Status in 1,000 Patients." Supportive Care in Cancer 8 (3): 175-9.

[23] Kirkova, J., et al. 2010. "Symptom Severity and Distress in Advanced Cancer." Palliative Medicine 24 (3): 330-9.

[24] Harding, R., et al. 2012. "Prevalence, Burden, and Correlates of Physical and Psychological Symptoms Among HIV Palliative Care Patients in Sub-Saharan Africa: An International Multicenter Study." Journal of Pain and Symptom Management 44 (1): 1-9.

[25] Schuit, K. W., et al. 1998. "Symptoms and Functional Status of Patients with Disseminated Cancer Visiting
Outpatient Departments." Journal of Pain and Symptom Management 16 (5): 290-7.

[26] Van den Beuken-van Everdingen, M., et al. 2007. "Prevalence of Pain in Patients with Cancer: a Systematic Review of the Past 40 Years." Annals of Oncology 18 (9): 1437-49.

[27] Potter, J., et al. 2003. "Symptoms in 400 Patients referred to Palliative Care Services: Prevalence and Patterns." Palliative Medicine 17 (4): 310-4.

[28] Murray, S. A., et al. 2007. "Patterns of Social, Psychological, and Spiritual Decline toward the End of Life in Lung Cancer and Heart Failure." Journal of Pain and Symptom Management 34 (4): 393-402. Wright, E. P., et al. 2014. Social problems in oncology. British Journal of Cancer 87 (10): 1099-104. stattrek, Random Number Generator.

[29] Karus, D., et al. 2005. "Patient Reports of Symptoms and their Treatment at Three Palliative Care Projects Servicing Individuals with HIV/AIDS." Journal of Pain and Symptom Management 30 (5): 408-17.

[30] Caraceni, A., et al. 2002. "Pain Measurement Tools and Methods in Clinical Research in Palliative Care: Recommendations of an Expert Working Group of the European Association of Palliative Care." Journal of Pain and Symptom Management 23 (3): 239-55.

[31] Morita, T., et al. 2005. "Late Referrals to Specialized Palliative Care Service in Japan." Journal of Clinical Oncology 23 (12): 2637-44.

[32] Ahmed, N., et al. 2004. "Systematic Review of the Problems and Issues of Accessing Specialist Palliative Care by Patients, Carers and Health and Social Care Professionals." Palliative Medicine 18 (6): 525-42.

[33] Solano, J. P., Gomes, B., and Higginson, I. J. 2006. “A Comparison of Symptom Prevalence in Far Advanced Cancer, AIDS, Heart Disease, Chronic Obstructive Pulmonary Disease and Renal Disease." Journal of Pain and Symptom Management 31 (1): 58-69.

[34] Genberg, B. L., et al. 2009. "A Comparison of HIV/AIDS-related Stigma in Four Countries: Negative Attitudes and Perceived Acts of Discrimination towards People Living with HIV/AIDS." Social science \& medicine 68 (12): 2279-87.

[35] Maman, S., et al. 2009. "A Comparison of HIV Stigma and Discrimination in Five International Sites: The Influence of Care and Treatment Resources in High Prevalence Settings." Social science \& medicine 68 (12): 2271-8.

[36] Moadel, A., et al. 1999. "Seeking Meaning and Hope: Self-reported Spiritual and Existential Needs among an Ethnically-diverse Cancer Patient Population.” Psycho-Oncology 8 (5): 378-85.

[37] Selman, L., et al. 2012. "The "Spirit 8" Successfully 

HIV/AIDS Reffered for Palliative Care

Captured Spiritual Well-being in African Palliative Care: Factor and Rasch Analysis." Journal of clinical epidemiology 65 (4): 434-43.

[38] Lemieux, L., et al. 2004. "Sexuality in Palliative Care: Patient Perspectives." Palliative Medicine 18 (7): 630-7.

[39] Hordern, A. J., and Street, A. F. 2007. "Communicating about Patient Sexuality and Intimacy after Cancer: Mismatched Expectations and Unmet Needs." Medical Journal of Australia 186 (5): 224.

[40] Hordern, A. J., and Currow, D. C. 2003. "A Patient-centred Approach to Sexuality in the Face of Life-limiting Illness." Medical Journal of Australia 179
(6): S8.

[41] Hordern, A. 2008. "Intimacy and Sexuality after Cancer: a Critical Review of the Literature." Cancer nursing 31 (2): E9-E17.

[42] Brechtl, J. R., et al. 2001. "The Use of highly Active Antiretroviral Therapy (HAART) in Patients with Advanced HIV Infection: Impact on Medical, Palliative Care, and Quality of Life Outcomes." Journal of Pain and Symptom Management 21 (1): 41-51.

[43] Selman, L. E., et al. 2011. "Quality of Life among Patients Receiving Palliative Care in South Africa and Uganda: a Multi-centred Study." Health Qual Life Outcomes 9: 21. 